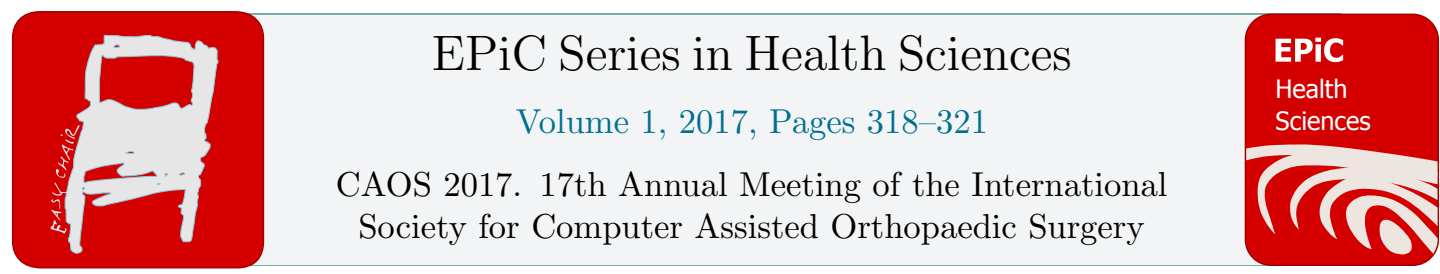

\title{
Trackerless 3D Ultrasound Stitching for Computer-Assisted Orthopaedic Surgery and Pelvic Fractures
}

\author{
Prashant Pandey ${ }^{1,2}$, Rafeef Abugharbieh ${ }^{2}$, Antony Hodgson ${ }^{1}$ \\ ${ }^{1}$ Surgical Technologies Laboratory, University of British Columbia, Vancouver, Canada, \\ ${ }^{2}$ Biomedical Signal and Image Computing Laboratory, University of British Columbia, Vancouver, \\ prashant@ece.ubc.ca, rafeef@ece.ubc.ca, ahodgson@mech.ubc.ca
}

\section{Introduction}

In pelvic fracture surgeries, percutaneous screws must be placed accurately for effective fixation and to prevent damage to surrounding tissue structures (Bates 2010). Fluoroscopy is currently used to image the pelvis to provide guidance, but this produces harmful ionizing radiation and does not allow three-dimensional (3D) visualisation (Gay 1992). Ultrasound offers three-dimensional, non-ionizing, real-time, and inexpensive imaging. It has thus emerged as an alternative to fluoroscopy for intraoperative imaging in computer-assisted orthopaedic surgery (CAOS). However, ultrasound-based surgical guidance is challenging because ultrasound produces inherently noisy images with limited field-of-view. While several techniques have been proposed to improve bone clarity in ultrasound scans (Hacihaliloglu 2009; Quader 2014), there is limited work on enhancing ultrasound's field-of-view for CAOS. In particular, improving the field-of-view for surgical guidance for pelvic fracture surgeries would be needed to achieve accurate and reliable registration to preoperative data, and accurate screw placement in the pelvis (Keil 2009).

In this work, we propose and evaluate the feasibility of a trackerless method for stitching volumetric ultrasound to achieve an extended field-of-view. Previous ultrasound stitching methods have used optical tracking to determine the spatial alignment between volumes (Dalvi 2010; Ni 2009), but these approaches require additional hardware. Our method contributes towards simpler surgical navigation as it does not require tracking equipment.

\section{Materials and Methods}

In this study, we obtained overlapping ultrasound volumes from a pelvic phantom, applied the proposed stitching method, and evaluated the accuracy of the extended volume against a computed tomography (CT) scan of the phantom (the ground-truth volume). 
Trackerless 3D Ultrasound Stitching for Computer-Assisted Orthopaedic Surgery ... P. Pandey et al.

The phantom consisted of a radio-opaque iliac crest from a human pelvis model (Sawbones, Vashon Island, WA, USA). The bone model was embedded in polyvinyl chloride gel and surrounded by 65 spherical steel fiducial markers ( $1 \mathrm{~mm}$ diameter). We used a SonixGPS ultrasound machine to acquire data using a motorized volumetric Ultrasonix 4DL14-5/38 transducer (BK Ultrasound, Peabody, MA, USA) transmitting at $6 \mathrm{MHz}$.

Three volumes were collected by manually moving the transducer along the phantom's length in the elevation direction. Neighbouring volumes had approximately $40 \%$ overlap. Each volume captured a $38 \times 40 \times 53 \mathrm{~mm}$ (lateral $\times a x i a l \times e l e v a t i o n a l)$ volume, represented by $473 \times 562 \times 91$ voxels.

Bone surfaces in the ultrasound were enhanced using confidence-map weighted phase symmetry (CPS). CPS identifies bone using local phase information and bone acoustic shadowing, resulting in increased bone signal-to-noise ratio (Quader 2014). CPS allows for the subsequent stitching process to use image features that strongly correspond to the bone surfaces, and is intended to increase robustness to noise in the ultrasound data.

We used a 3D implementation of the scale-invariant feature transform (SIFT) to extract features in the overlapping volume regions which are invariant to scale and orientation (Rister 2015). 3D SIFT feature extraction reduces errors in the subsequent point-based registration step because the output of CPS detection has a small-rate of false-positives and false-negatives (i.e. voxels wrongly identified as bone, and vice-versa) due to the 2D implementation of CPS. Figure 1 illustrates CPS detection and the advantage of using 3D SIFT feature extraction.

Feature extraction produced four point-sets (two-point sets per pair of neighbouring volumes). A coherent point drift (CPD) algorithm was used to rigidly register the point-sets and find alignment between volumes (Myronenko 2009). CPD uses a probabilistic framework to register point-sets and is robust to noise and outliers. The resulting transformations were applied to the raw ultrasound volumes in a global coordinate frame defined by the origin of the first volume. Overlapping regions between the volumes were combined using weighted averaging to create a smooth transition between neighbouring volumes. Figure 2 illustrates the stitched ultrasound volume and the CT-acquired volume.

The accuracy of the stitched volume was validated by measuring distances between fiducial markers in the stitched volume and comparing them to the true distances. The ground-truth measurements were acquired from a CT volume of the phantom. The absolute difference between these measured distances defined the error. Distances between fiducial markers were only measured if the markers were originally in two different ultrasound volumes. Additionally, surface registration was performed between the stitched ultrasound and ground-truth CT to assess the fidelity of the stitched bone surface, and the mean absolute surface distance and the Hausdorff distance between the two surfaces were calculated.

\section{Results}

Distances were measured between 12 pairs of fiducials. There was a mean error of $2.78 \mathrm{~mm}$ (standard deviation: $2.40 \mathrm{~mm}$ ) in the stitched ultrasound, which corresponds to a mean value of $5.1 \%$ of the true distances.

Surface registration resulted in a mean absolute surface distance of $0.54 \mathrm{~mm}$ (standard deviation: $0.45 \mathrm{~mm}$ ), and a Hausdorff distance of $2.61 \mathrm{~mm}$ from the stitched ultrasound to the ground-truth surface. 

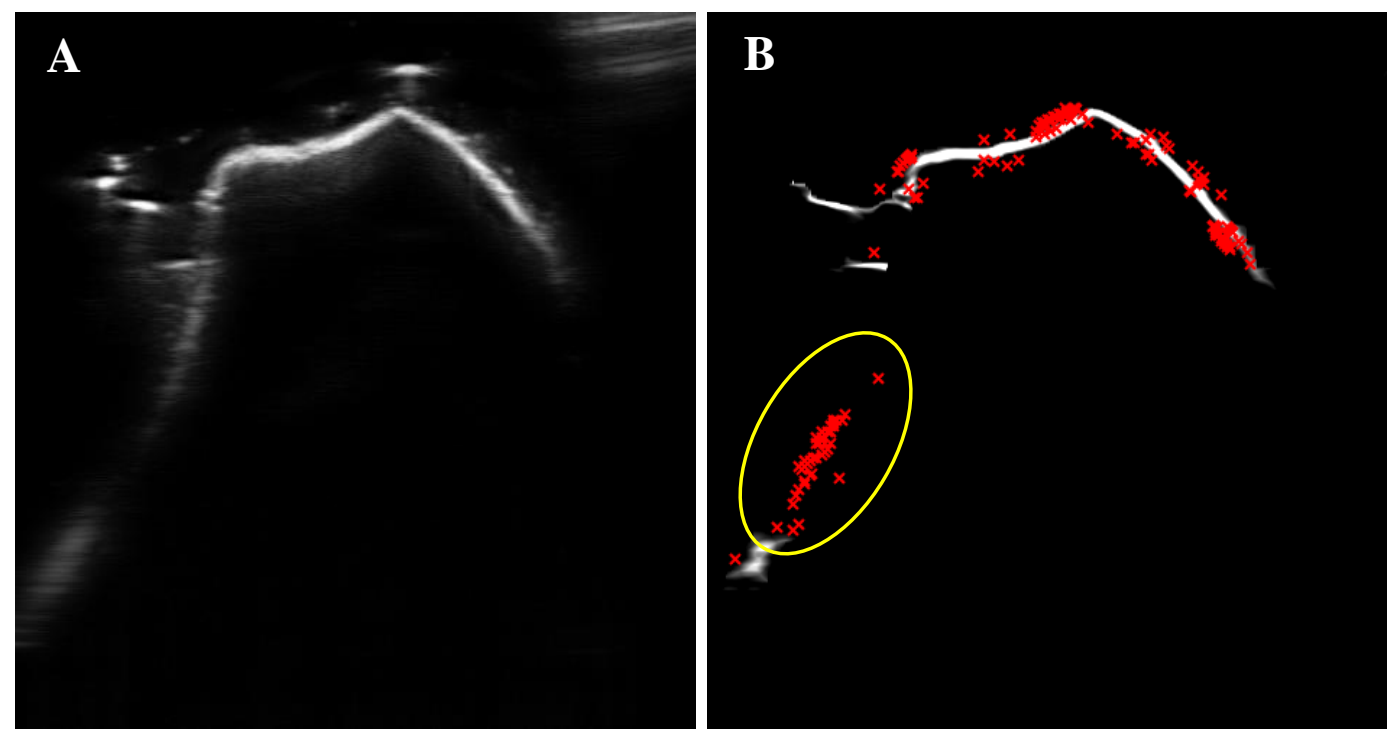

Figure 1: A: Raw ultrasound slice of the phantom showing the noisy bone surface and associated artefacts. B: Application of CPS and 3D SIFT to A results in accurate bone surface feature extraction. Crosses indicate extracted SIFT features. The oval highlights accurate feature extraction corresponding to the true bone surface.
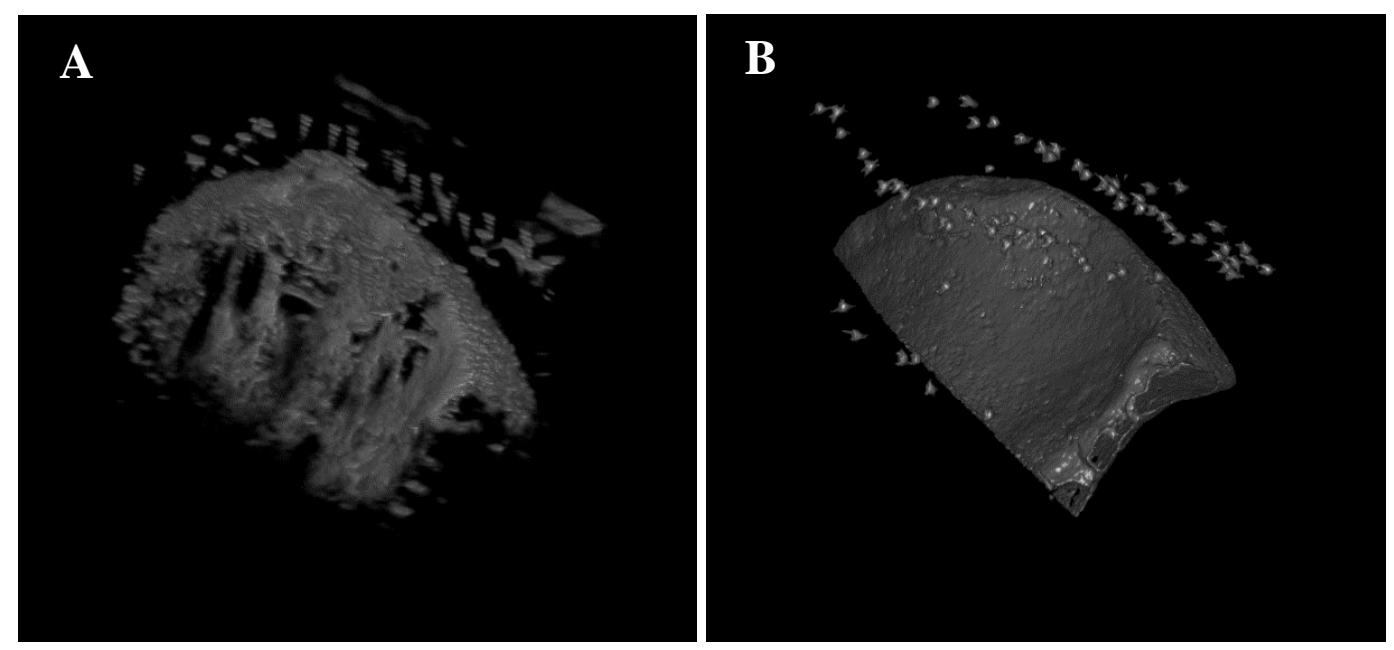

Figure 2: A: Final stitched ultrasound volume. B: CT-acquired ground-truth volume.

\section{Discussion}

This study proposed a trackerless framework for performing volumetric ultrasound stitching, based on 3D SIFT feature extraction and CPD-based registration. The method is designed for applications in CAOS, as bone is first localized in ultrasound using CPS detection. We succeeded in extending the field-of-view of 3D ultrasound by creating a $39 \times 43 \times 115 \mathrm{~mm}$ volume from three initial overlapping 
Trackerless 3D Ultrasound Stitching for Computer-Assisted Orthopaedic Surgery ... P. Pandey et al.

volumes, with reasonable overall accuracy. Our method can in principle be extended to an arbitrarily large volume, though the trade-off between size and accuracy would have to be considered based on the requirements for specific applications.

Our method does not rely on external tracking systems, yet performs comparably with the aforementioned studies. We show a mean post-registration surface error of $0.54 \mathrm{~mm}$, compared to $0.33 \mathrm{~mm}$ achieved by previous tracking-based stitching (Dalvi 2010). Our method achieved a mean distance error of $5.1 \%$, compared to $2 \%$ in a similar tracked 3D SIFT-based technique (Ni 2009).

We believe there is scope to further improve the stitching performance by introducing image features which better correspond with bone surfaces in ultrasound. Future work will also include validation of the method on clinical in vivo data and on a range of bone geometries.

\section{References}

Bates, P., Starr, A. \& Reinert, C., 2010. The Percutaneous Treatment of Pelvic and Acetabular Fractures, Bates, Starr and Reinert.

Dalvi, R., Hacihaliloglu, I. \& Abugharbieh, R., 2010. 3D ultrasound volume stitching using phase symmetry and harris corner detection for orthopaedic applications. In B. M. Dawant \& D. R. Haynor, eds. Medical Imaging. p. 762330. Available at: http://bisicl.ece.ubc.ca/focus/10_Rupin_SPIE.pdf.

Gay, S.B. et al., 1992. Percutaneous screw fixation of acetabular fractures with CT guidance: preliminary results of a new technique. American Journal of Roentgenology, 158(4), pp.819-822. Available at: http://www.ncbi.nlm.nih.gov/pubmed/1546599.

Hacihaliloglu, I. et al., 2009. Bone Surface Localization in Ultrasound Using Image Phase-Based Features. Ultrasound in Medicine \& Biology, 35(9), pp.1475-1487.

Keil, M. et al., 2009. Ultrasound and CT registration quality: Elastography vs. classical B-Mode. In 2009 IEEE International Symposium on Biomedical Imaging: From Nano to Macro. IEEE, pp. 967-970. Available at: http://ieeexplore.ieee.org/document/5193215/.

Myronenko, A. \& Song, X., 2009. Point-Set Registration: Coherent Point Drift. Advances in Neural Information Processing Systems 19, (September), pp.1009-1016. Available at: http://machinelearning.wustl.edu/mlpapers/paper_files/NIPS2006_613.pdf\%5Cnhttp://arxiv.org /abs/0905.2635\%5Cnhttp://dx.doi.org/10.1109/TPAMI.2010.46.

Ni, D. et al., 2009. Reconstruction of volumetric ultrasound panorama based on improved 3D SIFT. Computerized Medical Imaging and Graphics, 33(7), pp.559-566. Available at: http://linkinghub.elsevier.com/retrieve/pii/S0895611109000640.

Oszwald, M. et al., 2008. Accuracy of navigated surgery of the pelvis after surface matching with an amode ultrasound probe. Journal of Orthopaedic Research, 26(6), pp.860-864. Available at: http://doi.wiley.com/10.1002/jor.20551.

Quader, N., Hodgson, A. \& Abugharbieh, R., 2014. Confidence weighted local phase features for robust bone surface segmentation in ultrasound. MICCAI Workshop on Clinical Image-Based Procedures (CLIP), pp.76-83.

Rister, B. et al., 2015. Scale- and orientation-invariant keypoints in higher-dimensional data. In 2015 IEEE International Conference on Image Processing (ICIP). IEEE, pp. 3490-3494. Available at: http://ieeexplore.ieee.org/document/7351453/. 